\title{
Dynamics of laser-induced spin reorientation in $\mathrm{Co} / \mathrm{SmFeO}_{3}$ heterostructure
}

\author{
L. Le Guyader, A. Kleibert, and F. Nolting \\ Swiss Light Source, Paul Scherrer Institut, CH-5232 PSI-Villigen, Switzerland \\ L. Joly \\ IPCMS, 23 rue du Loess, BP 43 F-67034 Strasbourg Cedex 2, France
}

P. M. Derlet

Condensed Matter Theory Group, Paul Scherrer Institut, CH-5232 Villigen PSI, Switzerland

R. V. Pisarev

Ioffe Physical-Technical Institute, Russian Academy of Sciences, 194021 St. Petersburg, Russia

\author{
A. Kirilyuk, Th. Rasing, and A. V. Kimel \\ Radboud University Nijmegen, Institute for Molecules and Materials, 6525 AJ Nijmegen, The Netherlands ${ }^{5}$
}

(Received 5 November 2012; published 28 February 2013)

\begin{abstract}
Ultrafast control of a ferromagnet (FM) via exchange coupling with an antiferromagnet (AFM) is demonstrated in a $\mathrm{Co} / \mathrm{SmFeO}_{3}$ heterostructure. Employing time-resolved photoemission electron microscopy combined with x-ray magnetic circular dichroism, a sub-100-ps change of the Co spins orientation by up to $10^{\circ}$ driven by the ultrafast heating of the $\mathrm{SmFeO}_{3}$ orthoferrite substrate through its spin reorientation phase transition is revealed. Numerical modeling of the ultrafast-laser-induced heat profile in the heterostructure, and the subsequent coupled spins dynamics and equilibration of the spin systems suggest that the localized laser-induced spin reorientation is hindered compared with the static case. Moreover, numerical simulations show that a relatively small $\mathrm{Co} / \mathrm{SmFeO}_{3}$ exchange interaction could be sufficient to induce a complete and fast spin reorientation transition (SRT).
\end{abstract}

DOI: 10.1103/PhysRevB.87.054437

PACS number(s): 75.30.Kz, 75.50.Ee, 75.70.Cn, 75.78.Jp

\section{INTRODUCTION}

Ultrafast control of the magnetization of thin films using femtosecond laser pulses has attracted remarkable interest in the last fifteen years. Beginning with the report of an unexpected ultrafast demagnetization in a nickel thin film in $1996,{ }^{1}$ the research on ultrafast magnetization dynamics quickly developed. While the proper microscopic description of ultrafast demagnetization is still intensely debated, ${ }^{2}$ novel laser-induced magnetic phenomena have been discovered during these years in a large variety of materials ranging from ferromagnets (FMs) to antiferromagnets (AFMs) and from metals to insulators. ${ }^{3}$ While most of these studies where conducted in single-phase materials, exchange-coupled FM/AFM heterostructures are particularly interesting since novel material properties can there be engineered. Fundamentally, FM and AFM materials display very different magnetic properties. ${ }^{4}$ For example, in the quasistatic regime, FM materials react to moderate magnetic fields of the order of their anisotropy field $H_{A} \approx 1 \mathrm{~T}$ while, on the other hand, AFM materials are largely insensitive to magnetic fields up to the spin-flop transition. Combining these two types of materials in FM/AFM heterostructures offers the possibility of enhancing the magnetic anisotropy of the FM layer and creating a shifted hysteresis loop via the exchange bias effect, with numerous applications in spin-valve devices. ${ }^{5,6}$ In the dynamic regime, FM materials display a rather slow magnetic response given by their ferromagnetic resonance frequency $\omega \approx \gamma H_{A}$ of a few $\mathrm{GHz}$, while AFM materials have a much faster response thanks to their higher antiferromagnetic resonance frequency $\omega \approx$ $\gamma \sqrt{H_{\mathrm{ex}} H_{A}}$ of several hundreds of $\mathrm{GHz}$. This advantage has been recently employed to trigger ultrafast spin dynamics and spin reorientation in antiferromagnets. ${ }^{7-10}$ Combining these two different classes of materials in a FM/AFM heterostructure could thus produce a composite material with novel dynamical properties.

While there is plenty of literature on the static properties of such FM/AFM systems, their dynamical behaviours are often not considered. In particular, the possibility of speeding up the slow FM dynamics via coupling to the fast AFM dynamics seems to be very intriguing. Among the few studies that investigated this question, most of them considered the possibility of modifying the exchange bias with an optical pulse, such as in $\mathrm{NiFe} / \mathrm{NiO}$ by $\mathrm{Ju}$ et al., ${ }^{11-13}$ in $\mathrm{NiFe} / \mathrm{FeMn}$ by Weber et al., ${ }^{14,15}$ and in Co/IrMn by Dalla Longa et al. ${ }^{16,17}$ In all these cases, a reduction of the exchange bias within a few picoseconds followed by a few 100 ps recovery is reported. This sudden change of the exchange field and, in turn, the effective field, triggers damped spin precessions in the FM layer which are measured optically via the magneto-optical Kerr effect and which correspond to the relaxation of the FM spins towards the newly created equilibrium. While in principle, a sudden quenching of the exchange bias field could lead to a magnetization reversal (see Fig. 1 of Ref. 17), only weak effects of a few percent magnetization changes have so far been reported. Moreover, these triggered dynamics in the FM are of ferromagnetic nature since the AFM layer is used to pin the FM material and suddenly depin it upon laser excitation. By using stronger laser pulses, it is possible to bring the sample around its blocking temperature where changes in the AFM at the interface can be seen. ${ }^{18,19}$ However, the triggered FM dynamics in this case are again small in 
(a)

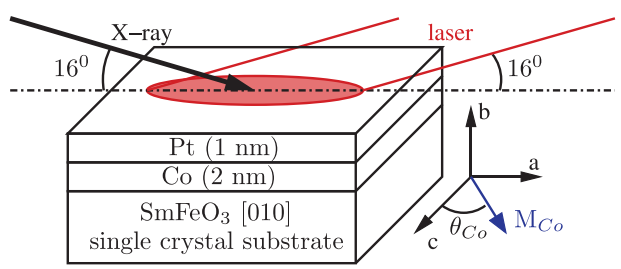

(b)
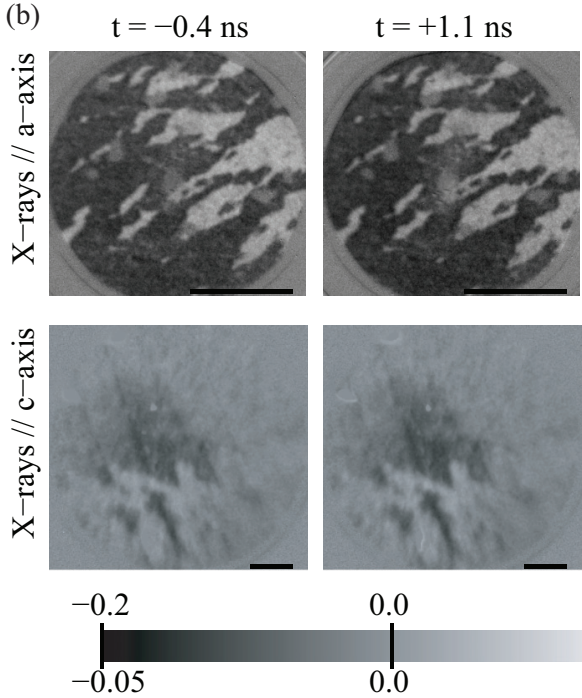
average $t_{>0}-t_{<0}$
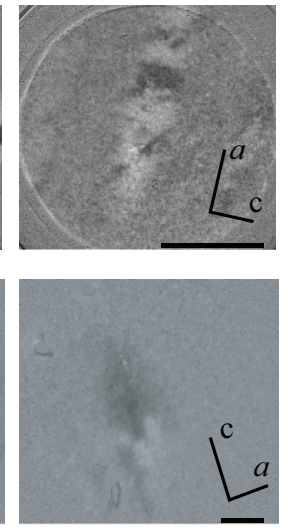

$+0.2 \mathrm{XMCD}$ +0.05 difference

FIG. 1. (Color online) (a) Sketch of various layers and orientation of sample with respect to $\mathrm{x}$-rays and laser-pulse propagation direction. The in-plane magnetization angle $\theta_{\mathrm{Co}}$ of the Co film is given with respect to the $\mathrm{SmFeO}_{3} c$ axis. (b) Time-resolved XMCD asymmetry images at the Co $L_{3}$ edge taken at two different time delays before $(t=-0.4 \mathrm{~ns})$ and after $(t=+1.1 \mathrm{~ns})$ the laser-pulse time overlap, as well as the averaged difference between images at positive $\left(t_{>0}\right)$ and negative $\left(t_{<0}\right)$ time delays of the measured sequences, for $\mathrm{x}$-rays propagating along the $a$ and the $c$ axis of the underlying $\mathrm{SmFeO}_{3}$ single crystal. In all images, the scale bars are $20 \mu \mathrm{m}$ long. The gray level values in the XMCD and difference images are given by the gray scales, respectively. The pump pulse was at $\lambda=800 \mathrm{~nm}$ wavelength with a pulse length equal to $\tau=50 \mathrm{fs}$ and fluence of $\mathcal{F}=10 \mathrm{~mJ} / \mathrm{cm}^{2}$.

amplitude and show slow relaxations by spin precessions towards the new equilibrium.

The question of whether or not it is possible to drive a FM at the speed of an AFM in a heterostructure cannot be addressed in those systems where the only possible action of the laser pulse is to reduce the coupling between the layers. This question can, however, be addressed in the $\mathrm{Co} / \mathrm{SmFeO}_{3}$ heterostructure where the quasistatic $90^{\circ}$ spin reorientation transition (SRT) in the rare-earth orthoferrite substrate induces a coupled SRT in the Co overlayer as demonstrated in Ref. 20. This coupled SRT in the Co overlayer indicates that the exchange coupling between the layers is preserved across the $15 \mathrm{~K}$ temperature range in which this SRT occurs.

In this paper we show that ultrafast laser heating of an orthoferrite substrate through its SRT results in an induced sub-100-ps spin reorientation of the exchange-coupled Co overlayer, this time limit being determined by the time resolution of the experiment. The amplitude of the observed exchange-induced spin reorientation in the Co layer is,

however, limited to about $10^{\circ}$ in contrast to the $90^{\circ}$ observed in the static case in this system. Different possible scenarios to explain this discrepancy were thus investigated. Effects from the inhomogeneous heating of the different layers by the laser pulse could be ruled out by numerical modeling of the laser-induced heat profile in the multilayer and by experiments at different laser pump wavelengths. The effect on the SRT of the formation of an exchange spring in the orthoferrite substrate between the hot surface and the cold bulk was investigated numerically and found to be significant but also to be overcome with higher pump fluence. Finally, the coupled dynamics of the orthoferrite and cobalt spins were simulated by numerical integration of a coupled pendulum equation and Landau-Lifschitz-Gilbert equation and found to be in good agreement with the experiments at the condition of a limited reorientation in the orthoferrite substrate. All these considerations suggest that the laser-induced dynamics in the orthoferrite itself is more complex than anticipated.

The paper is organized as follows: Section II is devoted to the description of the sample and of the experiments. In Sec. III, the obtained results are presented. In Sec. IV, the different simulations undertaken are described and consists of the modeling of the laser-heating profile in the multilayer in Sec. IV A, of the calculation of the transient quasi-equilibrium state of the heated sample in Sec. IV B, and finally of the simulation of the triggered exchange dynamics in Sec. IV C. Conclusions are presented in Sec. V.

\section{EXPERIMENTS}

The sample consists of a $2 \mathrm{~nm}$ magnetron sputtered Co film deposited on the [010]-oriented surface of a 1-mm-thick $\mathrm{SmFeO}_{3}$ single crystal substrate and capped with $1 \mathrm{~nm}$ $\mathrm{Pt}$ and is the same sample as in Ref. 20. The rare-earth orthoferrite $\mathrm{SmFeO}_{3}$ is a $G$-type canted antiferromagnet with an orthorhombically distorted perovskite structure. ${ }^{21}$ The Fe moments of this compound order antiferromagnetically below $673 \mathrm{~K}$, with the spins aligned along the $a$ axis of the crystal. Due to the Dzialoshinsky-Moria antisymmetric exchange interaction, the $\mathrm{Fe}$ spins are slightly canted by a small angle of $8.2 \times 10^{-3} \mathrm{rad}$, producing a net magnetization moment. $^{22}$ Due to this spin canting, the antiferromagnetic resonance splits in two modes without any applied magnetic field, resulting in a quasiferromagnetic mode at $270 \mathrm{GHz}$ and a quasi-antiferromagnetic mode at $550 \mathrm{GHz}$, both at room temperature. ${ }^{23}$ The $\mathrm{Sm}$ moments remain unordered down to $5 \mathrm{~K}$, below which they order antiferromagnetically with the Fe moments and induce a magnetization reversal. ${ }^{24}$ As the temperature is lowered from the Néel temperature, the Sm ions become increasingly polarized, resulting in a strongly temperature-dependent magnetic anisotropy for the $\mathrm{Fe}$ ions. This interaction between the Fe and Sm sublattices induces a second-order spontaneous spin reorientation transition (SRT), from above $495 \mathrm{~K}$ with the Fe spins aligned along the $a$ axis and the net magnetization along the $c$ axis to under $480 \mathrm{~K}$ with the Fe spins aligned along the $c$ axis and the net magnetization along the $a$ axis. The canting of the spins remains largely constant during the SRT. ${ }^{22}$ Recently, it has been reported that $\mathrm{SmFeO}_{3}$ is also a room-temperature improper ferroelectric and thus a multiferroic material, with a small ferroelectric 
polarization along the $b$ axis induced mostly by the inverse Dzialoshinsky-Moria exchange interaction. ${ }^{24}$ Considering that this ferroelectric polarization is induced by the magnetic order, and that its magnitude and direction does not change across the SRT, one can infer that its influence on the laser-induced spin dynamics investigated here should be negligible.

The $2 \mathrm{~nm}$ Co film sputtered on top of the $\mathrm{SmFeO}_{3}$ substrate is polycrystalline with a hexagonal-close-packed (hcp) structure $^{25}$ and couples ferromagnetically via an exchange interaction to the net moment of the $\mathrm{SmFeO}_{3}$ substrate. Thus, it displays the same SRT as the orthoferrite, i.e., a spontaneous reorientation of the Co moments from the substrate $a$ axis at low temperature to the $c$ axis at high temperature. ${ }^{20}$ Note that a magnetic dipolar coupling between the Co moments and the small $\mathrm{SmFeO}_{3}$ moments can be excluded since the observation of the induced Co SRT is very sensitive to the orthoferrite surface preparation. A change of magnetic anisotropy of the Co film induced by magnetostriction from the substrate can also be ruled out as the main coupling mechanism. While there is a small crystal elongation along the $c$ axis and contraction along the $a$ and $b$ axes of $\Delta l / l \approx 10^{-6}$ when heating through the SRT, ${ }^{26}$ the Co film with hcp structure displays a negative longitudinal magnetostriction, ${ }^{27-29}$ the effect of which counteracts the Co SRT instead of producing it. On the other hand, since a complete Co SRT is observed in this sample via slow heating, the magnetostriction is therefore negligible compared with the exchange coupling between the layers.

To image the magnetic domain configuration of the Co film, the Elmitec photoemission electron microscope (PEEM) at the Surface/Interface: Microscopy (SIM) beamline ${ }^{30}$ at the Swiss Light Source (SLS) was used. Employing the x-ray magnetic circular dichroism (XMCD) effect at the Co $L_{3}$ edge at $778 \mathrm{eV}$, a quantitative determination of the Co spin orientation angle can be derived. The intensity of a PEEM image of a magnetic sample recorded with circularly polarized x-rays is a spatially resolved measure of the total electron yield and can be written in the form of ${ }^{31}$

$$
I_{\sigma \pm}=I_{0}\left(1+\alpha \mathbf{M} \cdot \mathbf{L}_{ \pm}\right),
$$

where $I_{0}$ is the image intensity, $\alpha$ accounts for the magnitude of the XMCD effect, $\mathbf{M}$ is the magnetization vector, and $\mathbf{L}_{ \pm}$ is the X-ray polarization vector. An XMCD asymmetry image is obtained by a pixel-wise computation of $I_{\mathrm{XMCD}}=\left(I_{\sigma^{+}}-\right.$ $\left.I_{\sigma^{-}}\right) /\left(I_{\sigma^{+}}+I_{\sigma^{-}}\right)$which simplifies to $I_{\mathrm{XMCD}}=\alpha M \cos (\beta)$ where $\beta$ is the angle between the magnetization vector and the $\mathrm{X}$-ray polarization vector. This XMCD asymmetry image contains only normalized magnetic contrast information and typically shows white or black regions which are magnetic domains with magnetizations of opposite directions with respect to the $\mathrm{x}$-ray propagation vector. Assuming that the magnitude of the Co magnetization does not change significantly around the spin reorientation transition because of its much higher Curie temperature, a derivation of the Co magnetization angle is possible. It is convenient to choose the orthoferrite substrate $c$ axis as a reference axis for measuring angles, as shown in Fig. 1(a). The XMCD asymmetry image intensity becomes $I_{\mathrm{XMCD}}=\alpha M \cos \left(\theta_{c}-X_{c}\right)$, where $\theta_{c}$ is the angle of the Co magnetization $\mathbf{M}$ and $X_{c}$ is the angle of the x-ray direction. Defining the magnetic contrast $\xi$ as the difference between the
XMCD asymmetry value for white and black domains, the Co magnetization angle $\theta_{c}$ can be calculated via

$$
\theta_{c}=\arccos \left(\frac{\xi}{2 \alpha M}\right)+X_{c},
$$

where the quantity $2 \alpha M=0.32$ is a normalization constant which is determined experimentally from measurements of the magnetic contrast $\xi$ below the SRT in which case $\theta_{c}$ is known.

Time-resolved measurements of the Co magnetization configuration were performed by taking advantage of the pulsed nature of the X-rays produced by the SLS synchrotron via the gating of the detection in synchronization to an isolated $\mathrm{x}$-ray pulse present in the gap of the filling pattern of the storage ring. This scheme, presented in detail in Ref. 32, allows stroboscopic pump-probe imaging of the sample with a time resolution determined by the $70 \mathrm{ps}$ full width at half maximum (FWHM) temporal x-ray pulse length. To investigate the effect of the laser-induced heat profile on the achieved amount of spin reorientation, the wavelength of the pump laser was varied. For experiments with $\lambda=800 \mathrm{~nm}$ or $400 \mathrm{~nm}$ laser wavelength, an XL-500 oscillator from Femtolasers Produktions $\mathrm{GmbH}$ was used, producing a $\tau=50$ fs laser pulse with $500 \mathrm{~nJ}$ per pulse at a $5.2 \mathrm{MHz}$ repetition rate. This repetition rate is then reduced by a Pockels cell in combination with a crossed polarizer to match the $1.04 \mathrm{MHz}$ repetition rate of the isolated $\mathrm{X}$-ray probe pulses. The $400 \mathrm{~nm}$ pump was then obtained from the $800 \mathrm{~nm}$ fundamental wavelength by doubling with a $\beta$-barium-borate crystal with a conversion efficiency of $20 \%$. The $532 \mathrm{~nm}$ wavelength, $\tau=10$-ps-long pump was produced by a Duetto laser system from Time-Bandwidth Products AG with a maximum energy per pulse of $120 \mathrm{~nJ}$ used in the experiments. In all cases, the linearly p-polarized laser pump pulses were focused on the sample at a grazing incidence of $16^{\circ}$ as shown in Fig. 1(a) to a spot size of about $30 \times 100 \mu \mathrm{m}^{2}$ FWHM. The time overlap $(t=0)$ between the laser and the $\mathrm{X}$-ray pulse is unambiguously determined to better than \pm 15 ps by the sudden space charging ${ }^{33,34}$ which is induced by the laser pump pulse and which reduces significantly the amount of photoemitted electrons collected by the microscope. Finally, the sample can be heated via a resistive heater and the temperature measured with a thermocouple attached to the sample holder.

\section{RESULTS}

In order to study the $\mathrm{Co}$ spin dynamics in a $\mathrm{Co} / \mathrm{SmFeO}_{3}$ heterostructure upon heating the orthoferrite substrate through its SRT with a laser pulse, element-specific stroboscopic timeresolved XMCD PEEM measurements were conducted. The obtained time-resolved XMCD asymmetry images at the Co $L_{3}$ edge taken at two different time delays before $(t=-0.4 \mathrm{~ns})$ and after $(t=+1.1 \mathrm{~ns})$ the laser pulse, and for $\mathrm{x}$-rays propagating along the $a$ and $c$ axis of the underlying $\mathrm{SmFeO}_{3}$ substrate, are shown in Fig. 1(b). In these images, the Co magnetic domain configuration with typical sizes of the order of few micrometers can be seen. It must be noted that the sample position is different between the experiments with x-rays along the $a$ and $c$ axis and therefore the magnetic domains do not correlate between these two sets of images. In both cases, the temperature of the sample was adjusted by resistive heating 
such that before the laser pulse, the $\mathrm{Co} / \mathrm{SmFeO}_{3}$ heterostructure is already within the SRT temperature range. This explains why unsaturated magnetic domains are visible for $\mathrm{X}$-rays propagating both along the $a$ and $c$ axis of the underlying $\mathrm{SmFeO}_{3}$ substrate. It should be noted that a starting point within the SRT temperature range is a necessary requirement for this stroboscopic pump-probe measurement, since in principle, this SRT can occur via two equivalent routes as the net orthoferrite moment can reorient in opposite direction from the $a$ axis towards the $c$ axis (i.e., $+c$ or $-c$ ). Applying an in-plane magnetic field would break this equivalence of the reorientation routes. However, this would possibly alter the measured dynamics and substantially deviate the photoemitted electrons away from the microscope, rendering the measurement difficult. A more elegant approach would be to use circularly polarized laser pump pulses to break this equivalence of the two reorientation routes via the inverse Faraday effect as recently demonstrated. ${ }^{35}$ This, unfortunately, seems to be inefficient at the grazing incidence used in our setup. Instead, the equivalence between the two reorientation routes is broken here by heating the substrate within its SRT temperature range such that before the laser pulse, the coupled orthoferrite and Co SRT has already started. By comparing in both cases the domain configuration and magnetic contrast $\xi$ before $(t=-0.4 \mathrm{~ns})$ and after $(t=+1.1 \mathrm{~ns})$ the laser pulse in Fig. 1(b), it is evident that the domain configuration does not change, as expected, but also that the change in magnetic contrast within a domain is small.

To highlight this laser-induced change, an averaged image difference is computed, where from the sequences of timeresolved XMCD images measured at different time delays, the images taken after the laser pulse $t_{>0}$ are counted as positive and the images taken before the laser pulse $t_{<0}$ are counted as negative. In such difference images, as shown in Fig. 1(b), a region where the magnetic domain structures are again visible can be seen. The shape of this region is an elongated ellipse which is the signature of the focused laser beam impinging at grazing incidence on the sample surface. Note that, depending on the field of view used, the orientation of this ellipse is seen with a different orientation direction in the image frame. What is visible within the laser spot in the difference image thus corresponds to a localized change of magnetic contrast induced by the ultrafast laser pulse. By convention, the white color corresponds to positive values and the black color to negative values of $I_{\mathrm{XMCD}}$. In the case of X-rays propagating along the $a$ axis, the magnetic contrast displayed before the time overlap and in the difference image are opposite in sign since what appears as white domains, i.e., positive values, in the XMCD asymmetry image before the laser overlap turns into black domains, i.e., negative values, in the difference image. This means that the magnetic contrast is reduced after the laser heat pulse. On the contrary, in the case of x-rays along the $c$ axis, the magnetic contrast has the same sign before and in the difference images, meaning an increase of contrast after the laser heat pulse. This increase of contrast along the $c$ axis and decrease of contrast along the $a$ axis are better visualized in Fig. 2 where the magnetic contrast $\xi$ measured for domains inside the laser spot are shown as a function of the time delay after the laser pump pulse. Here again, a clear reduction of magnetic contrast along the $a$ axis and increase along the $c$ axis is observed. While in principle the reduction of magnetic

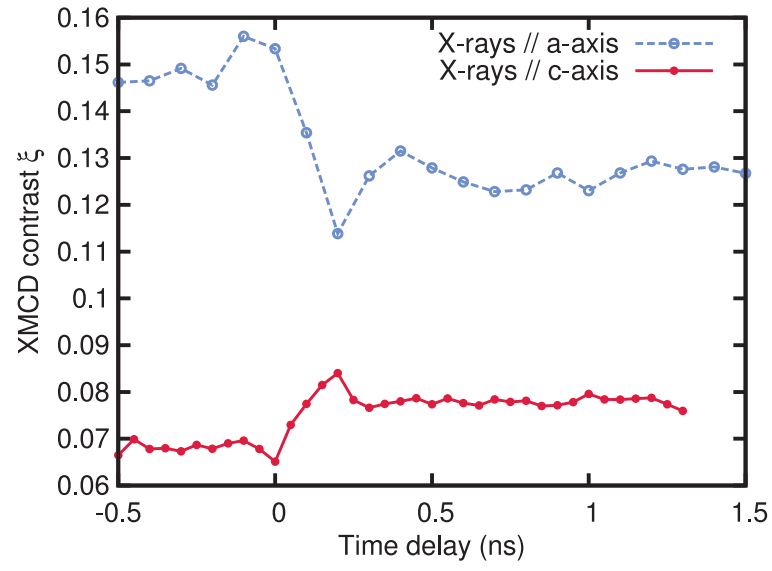

FIG. 2. (Color online) Magnetic contrast $\xi$ measured at Co $L_{3}$ edge for x-rays propagating along the $a$ and $c$ axes of the underlying $\mathrm{SmFeO}_{3}$ single crystal. The pump pulse was at $\lambda=$ $800 \mathrm{~nm}$ wavelength with $\tau=50 \mathrm{fs}$ pulse length and a $\mathcal{F}=10 \mathrm{~mJ} / \mathrm{cm}^{2}$ fluence.

contrast along the $a$ axis could be qualitatively explained by various effects other than a coupled SRT, the observation of an increased magnetic contrast for X-rays along the $c$ axis permits us to exclude them. Therefore, what is shown in Fig. 2 is not related with a partial demagnetization of the Co film by heating towards its Curie temperature as well as a decoupling between the $\mathrm{Co}$ and the $\mathrm{SmFeO}_{3}$ spins since, for these two effects, the same reduction of contrast would be observed with $\mathrm{X}$-rays propagating along the $a$ and $c$ axes. This increase of contrast along the $c$ axis and reduction along the $a$ axis is thus a clear signature of a spin reorientation in the Co film triggered by a laser pulse absorbed in the $\mathrm{SmFeO}_{3}$ substrate.

Assuming now that the only source of change in the magnetic contrast arises from the reorientation of the Co spins, it is then possible to use Eq. (2) to derive the Co spin angle $\theta_{\mathrm{Co}}$ from the orthoferrite $c$ axis as defined in Fig. 1(a). The Co spin angle change $\Delta \theta_{\mathrm{Co}}=\theta_{\mathrm{Co}}(t)-\theta_{\mathrm{Co}}\left(t_{<0}\right)$ induced by the laser from the negative time delay orientation is shown in Fig. 3. A sudden change of the Co spin angle is seen right after the laser overlap and corresponds to an alignment of the Co spins further along the $\mathrm{SmFeO}_{3} c$ axis in both measurements. Apart from the different amplitudes of reorientation in these two different measurements which are related to different experimental conditions, the Co spin dynamics is very similar in both cases and could be described as an overdamped spin precession around a new equilibrium. Together with the experimental data, the calculated response from the simulated coupled spin dynamics derived in Sec. IV C is shown as a continuous line where the simulated Co spin dynamics has been projected on the x-ray wave vector and convoluted by the x-ray pulse length of $70 \mathrm{ps}$. The similarity between the measured and the calculated reorientation indicates that the observed dynamics occurs within 100 ps. The amplitude of the Co reorientation obtained is, however, much smaller than the observed $90^{\circ}$ from the static case. ${ }^{20}$

To verify whether this initial quick and small-amplitude response of the Co spins is further followed by a larger and slower reorientation dynamics, measurements at a longer time scale were performed as well and the results are shown in Fig. 4 


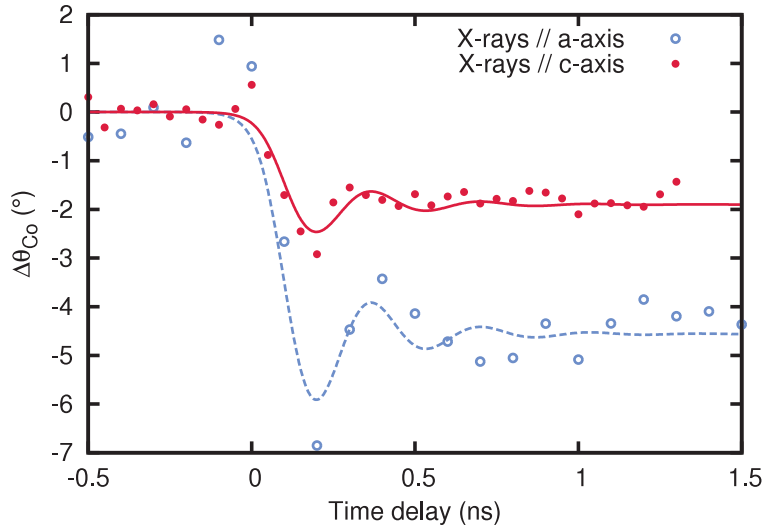

FIG. 3. (Color online) Co spin reorientation observed with $\mathrm{x}$-rays propagating along the $a$ and $c$ axes of the underlying $\mathrm{SmFeO}_{3}$ single crystal. The pump pulse was at $\lambda=800 \mathrm{~nm}$ wavelength with $\tau=$ 50 fs pulse length and a $\mathcal{F}=10 \mathrm{~mJ} / \mathrm{cm}^{2}$ fluence. The dots correspond to the measurement points and the lines to a simulated coupled-spin dynamics response convoluted by the temporal length of the x-ray probe.

for delays up to $15 \mathrm{~ns}$. Here, only a slow relaxation towards the initial orientation of the Co film is visible and is compatible with a slow cooling down of the $\mathrm{SmFeO}_{3}$ substrate, inducing a coherent rotation of the $\mathrm{SmFeO}_{3}$ and Co spins together back to the initial state before the laser pulse. Thus, there is no indication of a larger SRT occurring at longer time delays.

In order to investigate the effect of different levels of absorption of the pump laser pulse in the $\mathrm{SmFeO}_{3}$ substrate, experiments with different pump wavelengths were performed. The results shown in Fig. 5 represent the maximum amount of spin reorientation observed in the Co film that could be reached for each situation. Here again, despite a visible improvement for the case of $400 \mathrm{~nm}$ pump wavelength, the observed reorientation remains small compared to the $90^{\circ}$ obtained in the static case. ${ }^{20}$

Finally, time-resolved measurements performed as a function of the sample base temperature are shown in Fig. 6 while the laser fluence was kept constant. As the thermocouple with

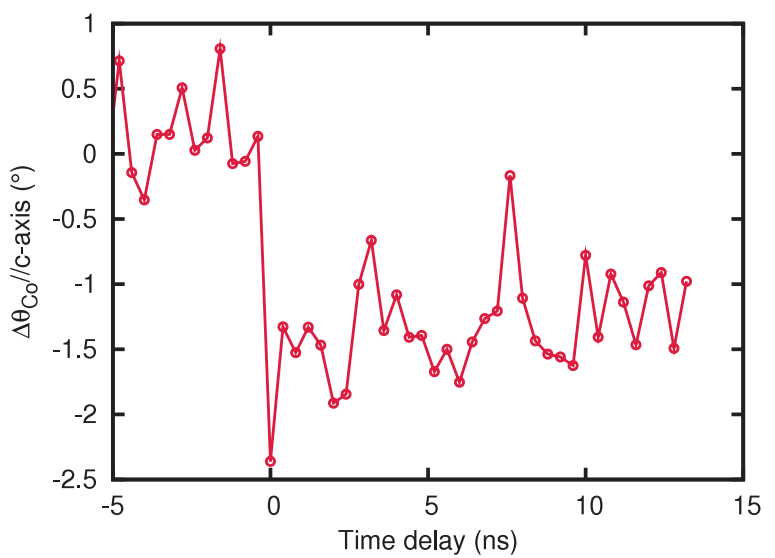

FIG. 4. (Color online) Time-resolved Co spin reorientation observed with $\mathrm{x}$-rays propagating along the $c$ axis for time delays up to $15 \mathrm{~ns}$. The pump pulse was at $\lambda=800 \mathrm{~nm}$ wavelength with $\tau=50 \mathrm{fs}$ pulse length and a $\mathcal{F}=10 \mathrm{~mJ} / \mathrm{cm}^{2}$ fluence.

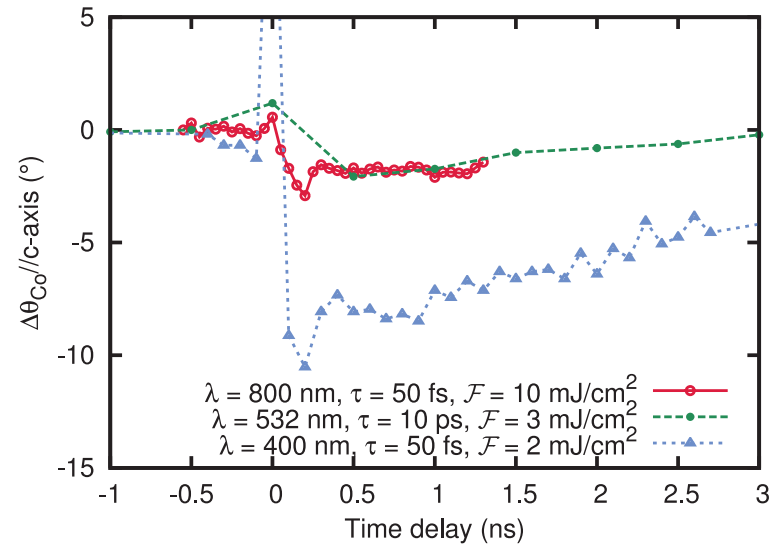

FIG. 5. (Color online) Time-resolved Co spin reorientation measured with x-rays propagating along the $c$ axis as a function of the laser wavelength.

which the sample temperature is measured is at a certain distance from the imaged region, the temperature measured is somewhat lower than the actual sample temperature. One can see that, starting closer to the end of the SRT at $383 \mathrm{~K}$, the only laser-induced effect visible is a reduction of the magnetic contrast. As the X-rays are propagating along the $c$ axis, this would translate in this geometry into a short-lived increase of the Co spin angle which would therefore go against the SRT. As the average temperature of the sample decreases, the laser-induced effect changes slowly to a longer-lived increase of magnetic contrast, i.e., a reduction of the Co spin angle as the SRT would produce. The short-lived effect is thus interpreted as a Co partial demagnetization which appears on top of the SRT, indicating that the laser-induced heating is significant.

The experimental findings regarding the Co spin dynamics subsequent to an ultrafast heating of the $\mathrm{Co} / \mathrm{SmFeO}_{3}$ heterostructure can be summarized as follows: First of all, a sub-100-ps reorientation of the Co spins takes place, followed by a slow relaxation back to the initial state. The absence of additional long-term dynamics apart from the relaxation means that the substrate has reached a transient quasi-equilibrium state within this 100 ps. In all the experiments, the amount of

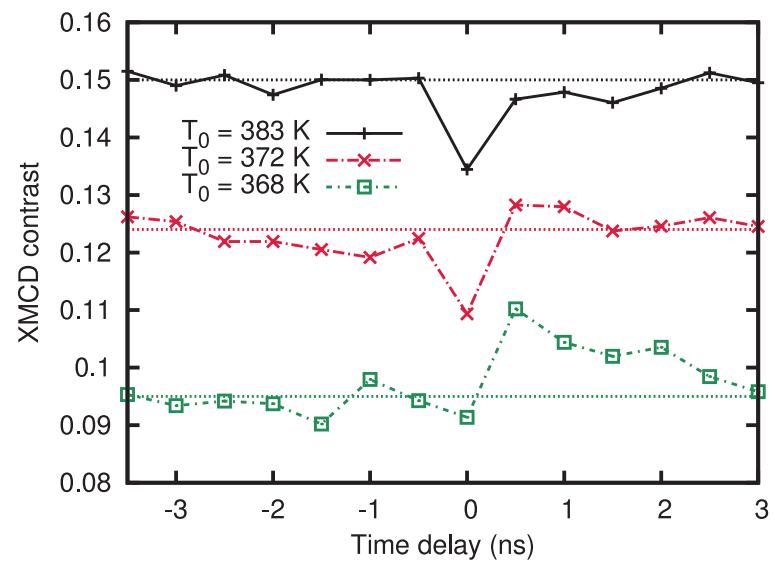

FIG. 6. (Color online) Time-resolved Co XMCD contrast measured with $\mathrm{x}$-rays propagating along the $c$ axis as a function of the base temperature $T_{0}$ of the sample. The pump pulse was at $\lambda=532 \mathrm{~nm}$ wavelength with $\tau=10 \mathrm{ps}$ pulse length and a $\mathcal{F}=3 \mathrm{~mJ} / \mathrm{cm}^{2}$ fluence. 
reorientation achieved was significantly smaller than the $90^{\circ}$ obtained in the static case, while the laser fluences used were enough to induce a transient partial demagnetization of the Co film.

\section{NUMERICAL MODELLING}

To better understand our experimental observations and the physics that they contain, detailed simulations were carried out. These simulations are divided in three different parts, which will be presented in the next sections. First of all, it is necessary to calculate the heat profile created in the sample by the absorption of the pump laser pulse in the various layers. After this, the transient quasi-equilibrium state of the inhomogeneously heated sample is determined. Finally, a simplified dynamics of an exchange-coupled Co-orthoferrite system is investigated.

\section{A. Laser-induced heat profile}

The laser intensity inside the $\mathrm{Pt} / \mathrm{Co} / \mathrm{SmFeO}_{3}$ multilayer was calculated using a matrix formalism of light scattering at the different interfaces and of light propagation inside the layers based on Abeles's formulas. ${ }^{36}$ From this laser intensity, the differential absorbance $d A(y)$ at any given depth $y$ from the sample surface can be derived [see Eq. (46) of Ref. 36]. The inhomogeneous temperature change induced by the absorption of the laser-pulse energy is then determined by the heat diffusion equation:

$$
\rho C_{\mathrm{p}} \frac{\partial T(y, t)}{\partial t}-k \nabla^{2} T(y, t)=I(t) d A(y),
$$

where $\rho$ is the density, $C_{\mathrm{p}}$ is the heat capacity, and $k$ is the heat diffusion of the materials and all are a function of the depth $y$ within the sample, and $I(t)$ is the time-dependent incoming laser intensity at the center of the laser spot. Neglecting any heat-diffusion effects, i.e., $k=0$ for all depths $y$, the temperature increase due to laser absorption is simply given by $\Delta T(y)=\mathcal{F} d A(y) /\left(\rho C_{\mathrm{p}}\right)$, where $\mathcal{F}$ is the incoming laser fluence. The values for the material parameters used are given in Table I. Three different cases have been simulated, corresponding to the three different laser wavelengths available in the experiments. The laser fluence used in these simulations has been adjusted for each wavelength such that the temperature increase at the surface of the $\mathrm{SmFeO}_{3}$ crystal is always $16 \mathrm{~K}$, which should be sufficient to induce a complete SRT. These laser fluences are $\mathcal{F}=0.35 \mathrm{~mJ} / \mathrm{cm}^{2}$ for $\lambda=400 \mathrm{~nm}$, $\mathcal{F}=3.20 \mathrm{~mJ} / \mathrm{cm}^{2}$ for $\lambda=532 \mathrm{~nm}$, and $\mathcal{F}=392 \mathrm{~mJ} / \mathrm{cm}^{2}$ for $\lambda=800 \mathrm{~nm}$. Comparing these values with those used in the experiments shows that the fluence was around 10 times higher

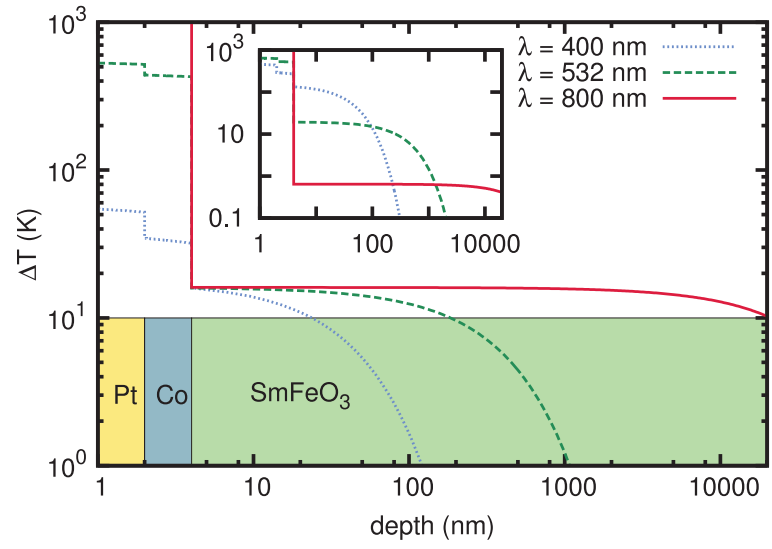

FIG. 7. (Color online) Heat profiles corresponding to a temperature increase of $\Delta T=16 \mathrm{~K}$ at the $\mathrm{SmFeO}_{3}$ surface for different laser wavelengths. The incident laser fluences used in the calculations were $\mathcal{F}=0.35 \mathrm{~mJ} / \mathrm{cm}^{2}$ for $\lambda=400 \mathrm{~nm}, \mathcal{F}=3.20 \mathrm{~mJ} / \mathrm{cm}^{2}$ for $\lambda=532 \mathrm{~nm}$, and $\mathcal{F}=392 \mathrm{~mJ} / \mathrm{cm}^{2}$ for $\lambda=800 \mathrm{~nm}$. The heat profiles for the experimental fluences of $\mathcal{F}=2 \mathrm{~mJ} / \mathrm{cm}^{2}$ for $\lambda=400 \mathrm{~nm}$, $\mathcal{F}=3 \mathrm{~mJ} / \mathrm{cm}^{2}$ for $\lambda=532 \mathrm{~nm}$, and $\mathcal{F}=10 \mathrm{~mJ} / \mathrm{cm}^{2}$ for $\lambda=800 \mathrm{~nm}$ are shown in the inset.

for $400 \mathrm{~nm}$ wavelength, about the same for $532 \mathrm{~nm}$ wavelength, and 40 times lower for $800 \mathrm{~nm}$ wavelength than the fluence required in the simulation to obtain a $\Delta T=16 \mathrm{~K}$ temperature increase at the $\mathrm{SmFeO}_{3}$ surface. Shown in Fig. 7 are the heat profiles obtained from these simulations as a function of the depth $y$ from the sample surface. ${ }^{37}$

Starting with the 800-nm-wavelength case, the calculation in Fig. 7 shows that, due to the low absorption of the orthoferrite at this wavelength, ${ }^{38}$ the fluence required to obtain the proper temperature increase at the $\mathrm{SmFeO}_{3}$ surface in order to induce the full SRT is very high and brings the Co film way above its Curie temperature and even possibly destroys it. Since the ratio between the temperature increase in the Co film and at the $\mathrm{SmFeO}_{3}$ surface is independent of the incoming laser intensity, it is easy to calculate the maximum SRT achievable without completely demagnetizing the Co film. With a Co film heated to less than $10^{3} \mathrm{~K}$, the $\mathrm{SmFeO}_{3}$ surface is heated to less than $0.16 \mathrm{~K}$. Considering that a $90^{\circ}$ SRT occurs within a $10 \mathrm{~K}$ temperature change, this leads to a maximum expected effect of about $1^{\circ}$. It is thus clear that this large heat difference between the Co film and the $\mathrm{SmFeO}_{3}$ surface is a limiting factor in achieving a $90^{\circ} \mathrm{SRT}$ with a $\lambda=800 \mathrm{~nm}$ laser pump wavelength, which is a direct consequence of the low absorption in the orthoferrite at this wavelength.

TABLE I. Parameters used in the simulations for the layer thickness $d$, density $\rho$, heat capacity $C_{\mathrm{p}}$, and complex refractive index $\tilde{n}$ for the different wavelength $\lambda$ of the different materials constituting the sample.

\begin{tabular}{lcccccc}
\hline \hline & $d$ & $\rho$ & $C_{\mathrm{p}}$ & & \multicolumn{2}{c}{} \\
\cline { 5 - 7 } & $(\mathrm{nm})$ & $\left(10^{3} \mathrm{~kg} \mathrm{~m}^{-3}\right)$ & $\left(\mathrm{J} \mathrm{kg}^{-1} \mathrm{~K}^{-1}\right)$ & $\lambda=400 \mathrm{~nm}$ & $\lambda=532 \mathrm{~nm}$ & $\lambda=800 \mathrm{~nm}$ \\
\hline $\mathrm{Pt}$ & 1 & 21.45 & 130 & $1.718+2.84 i$ & $2.074+3.63 i$ & $2.839+4.95 i$ \\
$\mathrm{Co}$ & 2 & 8.90 & 420 & $1.455+3.00 i$ & $2.209+3.9 i$ & $3.618+4.71 i$ \\
$\mathrm{SmFeO}_{3}$ & $\infty$ & 7.26 & 453 & $2.5+0.7 i$ & $2.4+0.1 i$ & $2.3+0.0013 i$ \\
\hline \hline
\end{tabular}


To overcome this limitation, it is necessary to change the laser wavelength to higher photon energy for which the $\mathrm{SmFeO}_{3}$ absorption is increased. The resulting heat profiles obtained with $532 \mathrm{~nm}$ and $400 \mathrm{~nm}$ are shown in Fig. 7 and indeed display an increasingly more balanced heat distribution between the various layers as the photon energy is increased. For these wavelengths, it is then possible to heat the $\mathrm{SmFeO}_{3}$ such that a complete SRT is obtained while the Co overlayer stays well below the Curie temperature. While measurements with $400 \mathrm{~nm}$ clearly show an improvement in the amplitude of Co spin reorientation obtained, as shown in Fig. 5, it is still far from being complete. As the absorption in the $\mathrm{SmFeO}_{3}$ increases, the penetration depth of the laser pulse also decreases, leading to more surface rather than bulk heating of the $\mathrm{SmFeO}_{3}$. As shown in Fig. 7, in the case of the $400 \mathrm{~nm}$ pump wavelength, the temperature change drops within few tens of nanometers. One could therefore expect that the heated spins at the $\mathrm{SmFeO}_{3}$ surface are pinned by the cold bulk, forming an exchange spring inside the crystal which could severely hinder the $90^{\circ}$ SRT. It is thus necessary to calculate the spin configuration in the $\mathrm{SmFeO}_{3}$ for the case of an inhomogeneous heating created by the absorbed laser pump pulse.

\section{B. Transient state}

Simulation of the spin dynamics induced by the laser-pulse energy absorbed in the $\mathrm{SmFeO}_{3}$ substrate is not a trivial task since, to the best of our knowledge, no micromagnetic simulation code for inhomogeneously excited antiferromagnet has been demonstrated so far. While neglecting the actual dynamical nature of the spin reorientation, the equilibrium configuration that the spin would eventually reach, if the heat profile induced by the laser were permanent, is a much simpler problem. At the same time, such simulations would give some insights into the maximum amount of spin reorientation that can be reached given a certain laser-heat-induced profile. The actual equilibrium spin configuration is mainly the result of two competing energy terms, which are the temperaturedependent magnetic anisotropy energy, responsible for the actual SRT, and the exchange energy, which should prevent the reorientation to occur due to the coupling with the unheated cold bulk.

For the modeling, we neglect any other energy contributions as well as the Gaussian laser profile in the $x z$ plane, resulting in an effective one-dimensional problem, which is the depth $y$ within the crystal. In addition, we neglect any effect due to the coupling with the Co layer. We neglect any heat diffusion as well. This means that the spins have enough time to fully reorient to the new equilibrium configuration. All these approximations should maximize the amount of spin reorientation obtained and thus allow us to calculate its upper limit within these approximations.

In $\mathrm{SmFeO}_{3}$, the anisotropy energy $E_{a}$ can be written as ${ }^{39}$

$$
E_{a}(T)=\int_{-\infty}^{0}\left[K_{2}(T) \sin ^{2}(\theta)+K_{4} \sin ^{4}(\theta)\right] d y,
$$

where $K_{2}(T)$ is the second-order anisotropy constant, which varies linearly with the temperature in the SRT region, $K_{4}$ is the fourth-order anisotropy constant, which is independent

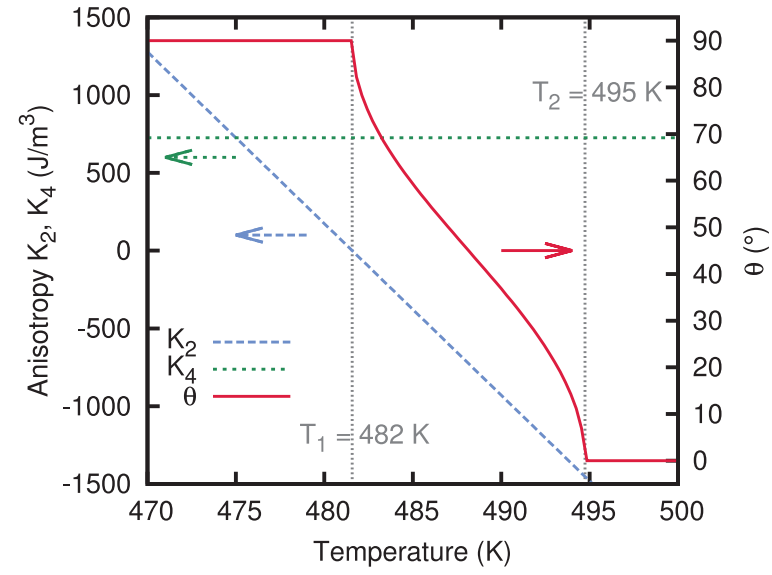

FIG. 8. (Color online) Anisotropy constant $K_{2}$ and $K_{4}$ and $\mathrm{SmFeO}_{3}$ magnetization angle $\theta$ with respect to the $c$ axis as function of the temperature within the SRT.

of the temperature in the SRT region, and $\theta(y)$ is the angle between the small $\mathrm{SmFeO}_{3}$ magnetization and the $c$ axis at the depth $y$ from the $\mathrm{SmFeO}_{3}$ surface. By minimizing the anisotropy energy with respect to $\theta$, one derives the equilibrium orientation of the magnetization as function of the temperature for an homogeneously heated sample. This gives the SRT shown in Fig. 8, based on the values for $K_{2}(T)$ and $K_{4}$ reported in the literature. ${ }^{40}$

In the case of inhomogeneous heating, the equilibrium configuration arising from the anisotropy term becomes position dependent and thus competes with the exchange energy term $E_{\mathrm{ex}}$, which can be written as

$$
E_{\mathrm{ex}}=\int_{-\infty}^{0} A\left(\frac{d \theta}{d y}\right)^{2} d y
$$

where the exchange stiffness constant $A=n J m_{0}^{2} / b=3.33 \times$ $10^{-11} \mathrm{~J} \mathrm{~m}^{-1}$ in which $n=4$ is the number of $\mathrm{Fe}$ ions per unit cell, $b=0.5592 \mathrm{~nm}$ is the lattice constant along the $b$ axis, ${ }^{41}$ and $J m_{0}^{2}$ is estimated via the mean-field relation $z J m_{0}^{2}=3 k_{b} T_{N}$ where $z=6$ is the coordination number, $T_{N}=674 \mathrm{~K}$ is the Néel temperature of the orthoferrite, and $k_{b}=1.381 \times 10^{-23} \mathrm{~J} \mathrm{~K}^{-1}$ is the Boltzmann constant, giving $J m_{0}^{2}=4.65 \times 10^{-21} \mathrm{~J}$.

Considering only the anisotropy and exchange energy, the thermodynamical potential is

$$
\Phi(\theta)=\int_{-\infty}^{0}\left[A\left(\frac{d \theta}{d y}\right)^{2}+K_{2}(T) \sin ^{2}(\theta)+K_{4} \sin ^{4}(\theta)\right] d y .
$$

Euler's equation of the variational problem of this functional is given by ${ }^{39}$

$$
\frac{d^{2} \theta}{d y^{2}}=\frac{K_{2}(T)}{A} \cos (\theta) \sin (\theta)+2 \frac{K_{4}}{A} \cos (\theta) \sin ^{3}(\theta) .
$$

Solving Eq. (7) numerically in the case of shallow energy landscape which occurs during the SRT is not trivial and leads to numerical instabilities with the usual approach of the initial-value problem when $\theta$ and $\theta^{\prime}$ at $y=-\infty$ are given. An alternative procedure is to solve the equation as a boundary 


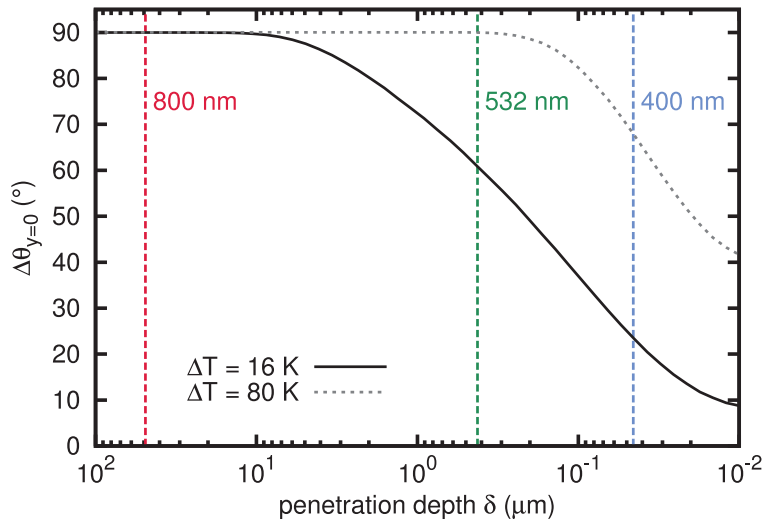

FIG. 9. (Color online) Amount of spin reorientation $\Delta \theta$ obtained at the $\mathrm{SmFeO}_{3}$ surface as function of the light-penetration depth $\delta$ for two different surface temperature changes $\Delta T$. The vertical lines indicate the $\mathrm{SmFeO}_{3}$ penetration depth for the indicated wavelengths.

value problem where $\theta(-\infty)$ and $\theta(0)$ are given and then to search for the resolved spin configuration minimizing the total energy as function of $\theta(0) .{ }^{42}$ In those calculations, the laser heating is described by $T(y)=T_{0}+\Delta T e^{\alpha y}$, where $T_{0}$ is the $\mathrm{SmFeO}_{3}$ temperature without laser heating, $\Delta T$ is the laserinduced temperature change at the surface, and $\alpha=4 \pi \kappa / \lambda$ is the optical absorption of the $\mathrm{SmFeO}_{3}$ in which $\kappa$ is the extinction coefficient from the complex index of refraction $\tilde{n}=n+i k$. Given a certain laser-induced temperature change $\Delta T$ at the surface, the amount of spin reorientation $\Delta \theta$ as a function of the light penetration depth $\delta=1 / \alpha$ can be calculated and is shown in Fig. 9 for two different temperature induced changes. In addition, the amount of spin reorientation $\Delta \theta$ as a function of depth from the $\mathrm{SmFeO}_{3}$ surface for the large temperature increase of $\Delta T=80 \mathrm{~K}$ is shown in Fig. 10 .

At very low absorption, such as when $\lambda=800 \mathrm{~nm}$, the penetration depth of the light is long enough to induce a quasihomogeneous heating such that no limitation of the amplitude of the spin reorientation from the exchange spring formation is to be expected. The limiting factor in this case is only the unbalanced heating between the Co film and the $\mathrm{SmFeO}_{3}$ substrate, as demonstrated in the previous section.

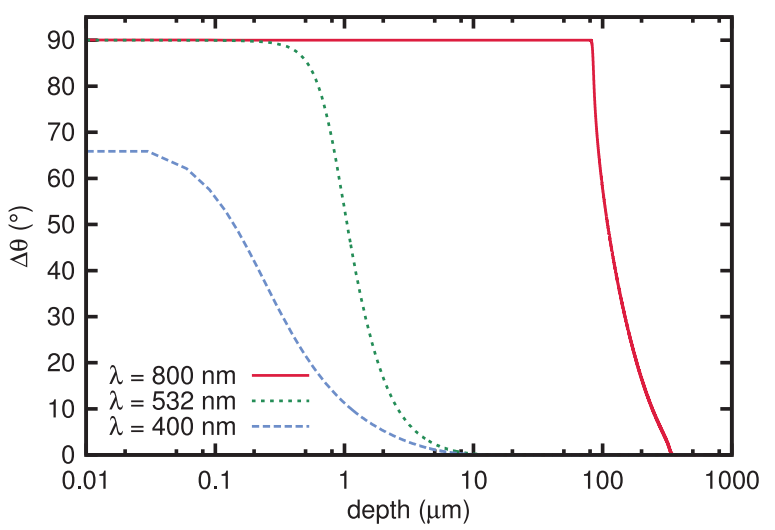

FIG. 10. (Color online) Spin reorientation $\Delta \theta$ as function of depth from the surface for different wavelengths in the case of a surface temperature change $\Delta T=80 \mathrm{~K}$.
At the opposite case of strong absorption, which corresponds to experiments with $\lambda=400 \mathrm{~nm}$ wavelength, the achievable SRT is found to be limited by the formation of an exchange spring with the cold bulk. Increasing the laser fluence, i.e., the temperature change at the $\mathrm{SmFeO}_{3}$ surface $\Delta T$, partly overcomes this pinning effect by heating deeper into the sample. However, a five-times increase of the laser fluence still leads to exchange spring effects, as shown by the $70^{\circ}$ reorientation obtained in this case. This is better visualized in the reorientation profile in Fig. 10 where this $70^{\circ}$ occurs only within the first $100 \mathrm{~nm}$ of the single crystal. Nevertheless, this achievable reorientation is much larger than what has been observed in the experiments shown in Fig. 5.

At intermediate absorption corresponding to experiments realized with $532 \mathrm{~nm}$ wavelength, a large spin reorientation can be expected even for moderate heating, and complete reorientation should be achieved for stronger heating. This finding is somewhat in conflict with the results shown in Fig. 6 where large heating effects are demonstrated by the transient demagnetization observed for base temperature at the end of the SRT, while small reorientation is observed when cooling down through the SRT.

The comparison of these calculations with the experiments strongly suggests that there must be another effect taking place which prevent the orthoferrite to fully reorient after laser heating. It should be noted that in fact a $90^{\circ}$ laser-induced spin reorientation in any rare-earth orthoferrite has not yet been experimentally demonstrated. The few studies done show a maximum $20^{\circ}$ reorientation..$^{35,43-45}$ It seems that ultrafast laser heating is not equivalent to slow heating. Such an effect has been reported in another system has well, ${ }^{46}$ where it was suggested that the laser pulse brings the system into a metastable state not accessible otherwise. While further experiments would be required to unveil this possible transient metastable state, it is nevertheless interesting to investigate the possible coupled-spin dynamics that could occur with a $90^{\circ}$ SRT.

\section{Coupled-spin dynamics}

To study how a thin film of cobalt might react to the spin dynamics of the underlying orthoferrite substrate, a number of simplifying assumptions are made. It is assumed that the Co moment interacts directly with the Fe moments via a nearest-neighbor ferromagnetic exchange mechanism, and the exchange field that the Co moments see is derived from the net magnetization of bulk orthoferrite - thus the orthoferrite is treated as an external field that is not affected by the presence of the cobalt and the associated interface geometry. The interaction term is, therefore,

$$
\mathcal{H}_{\text {Co, exchange }}=\frac{1}{2} J_{\mathrm{c}} \mathbf{m}_{\mathrm{c}} \cdot \mathbf{m} .
$$

In the above, $\mathbf{m}$ and $\mathbf{m}_{\mathrm{c}}$ are the total magnetic moments per unit cell of the orthoferrite and the cobalt, respectively, and $J_{\mathrm{c}}$ is their exchange interaction parameter. The factor of one half arises from the Co moment interacting with only two of the four sublattice magnetizations of the orthoferrite. Finally, the rigid moment approximation is considered for the Co system and thus spatial fluctuations in the magnetic moment within 
the Co layer are also not considered. These simplifications, whilst severe, make the problem theoretically tractable.

The Landau-Lifschitz-Gilbert (LLG) equation for the Co moment is

$$
\frac{d \mathbf{m}_{\mathrm{c}}}{d t}=-\frac{\gamma}{1+\alpha_{\mathrm{c}}^{2}}\left[\mathbf{m}_{\mathrm{c}} \times \mathbf{B}_{\mathrm{c}, \mathrm{eff}}-\frac{\alpha_{\mathrm{c}}}{m_{\mathrm{c}}} \mathbf{m}_{\mathrm{c}} \times\left(\mathbf{m}_{\mathrm{c}} \times \mathbf{B}_{\mathrm{c}, \mathrm{eff}}\right)\right],
$$

where $\gamma$ is the gyromagnetic ratio and $\alpha_{\mathrm{c}}$ is an empirical damping parameter for Co. Here the time-dependent effective field is

$$
\mathbf{B}_{\mathrm{c}, \mathrm{eff}}(t)=-\frac{d \mathcal{H}(t)}{d \mathbf{m}_{\mathrm{c}}}=-\frac{1}{2} J_{\mathrm{c}} \mathbf{m}(t),
$$

where the temporal evolution of the orthoferrite magnetization $\mathbf{m}(t)$ is entirely determined by the evolution of the orthoferrite AFM moment $\mathbf{l}(t)$ via

$$
\mathbf{m}(t) \simeq \frac{1}{2 J} \mathbf{l}(t) \times \mathbf{D} .
$$

In the above, $J$ is the exchange interaction parameter and D is the Dzyaloshinskii-Moriya interaction vector parameter for the orthoferrite. Equation (11) embodies the "slave" approximation to the magnetization ${ }^{45,47}$ in which the antiferromagnetic moment dynamics is well approximated via damped harmonic motion-the so-called pendulum approach. A derivation for the present context is given as supplementary material, ${ }^{48}$ which gives $\mathbf{l}(t)$ as a solution to a nonlinear damped harmonic oscillator [Eq. (22) in Ref. 48]. Equation (11) is an approximation to Eq. (19) in Ref. 48, which ignores the effect of the precessional dynamics of the AFM moment on the total moment. For the present system, this was found to be valid.

To include the expected thin film demagnetization field, an anisotropy term is added to the magnetic energy of the form

$$
\mathcal{H}_{\mathrm{SA}}=K_{\mathrm{SA}}\left[1-\left(\hat{\mathbf{m}}_{\mathrm{c}} \cdot \hat{\mathbf{e}}\right)^{2}\right],
$$

where the reference direction is defined as $\hat{\mathbf{e}}=(0,0,1)$ and $K_{\mathrm{SA}}=-\mu_{0} M_{\mathrm{c}}^{2} / 2$. Here $M_{\mathrm{c}}$ is the appropriate magnetization for Co. Taking the magnetization of bulk Co to be $M_{\mathrm{c}}=$ $1400 \times 10^{3} \mathrm{~A} / \mathrm{m}, K_{\mathrm{SA}}=-1.2 \times 10^{6} \mathrm{~J} / \mathrm{m}^{3}$, which corresponds to an effective demagnetization field magnitude $\mu_{0} M_{\mathrm{c}}$ of approximately $1.8 \mathrm{~T}$.

Figure 11 displays the three-dimensional evolution of the Co moment for the orthoferrite exchange field arising from the orthoferrite reorientations at the temperatures 460, 464, and $468 \mathrm{~K}$-simulated in Ref. 48 and shown in Fig. 1(a) in Ref. 48. The Co moment direction is initially orientated perpendicular to the AFM moment under the assumption that it initially aligns with the total magnetic moment of the orthoferrite. In Fig. 11, the left panels display the in-plane angular evolution and the right panels display the out-of-plane evolution. The damping term $\alpha_{c}$ is chosen to be 0.014 which is the known value for bulk Co. The upper panels of Figs. 11(a) and 11(b) correspond to an exchange interaction between the orthoferrite and the Co of $J_{\mathrm{c}} m_{0}=-0.1 \mathrm{~T}$, the middle panels (c) and (d), correspond to $J_{\mathrm{c}} m_{0}=-10 \mathrm{~T}$, and the lower panels (e) and (f) correspond to $J_{\mathrm{c}} m_{0}=-150 \mathrm{~T}$. The lower limit is that used in Sec. III to fit the experimental data, whereas the upper limit is comparable to
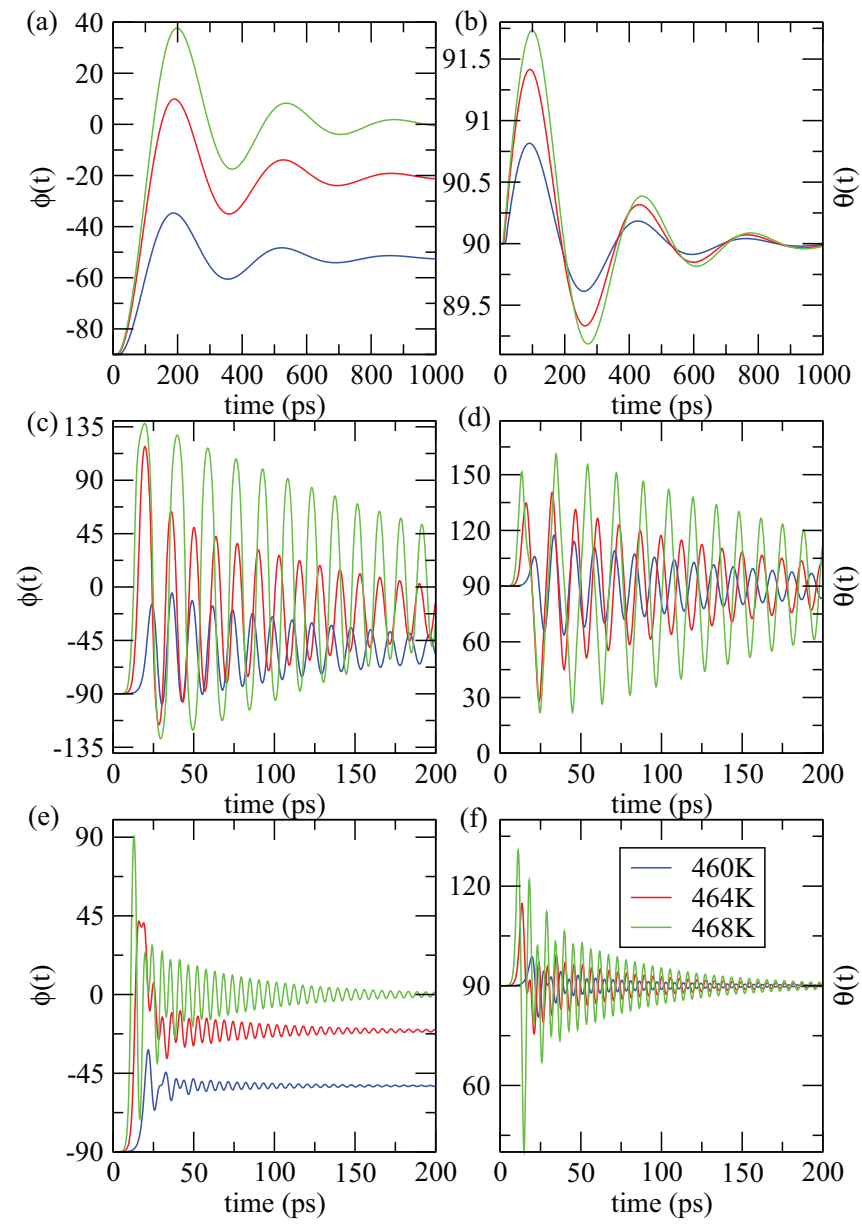

FIG. 11. (Color online) Angular evolution of the Co moment for the (left panels) in-plane and (right panels) out-of-plane component for three different values of $J_{\mathrm{c}} m_{0}$, where the upper-two panels are at $-0.1 \mathrm{~T}$, the middle two at $-10 \mathrm{~T}$, and the lower two at $-150 \mathrm{~T}$. In all cases the damping coefficient of the Co moment was set to $\alpha_{\mathrm{c}}=$ 0.014. For each figure, the three curves correspond to the anisotropy energy landscape corresponding to the temperatures 460, 464, and $468 \mathrm{~K}$.

the exchange interaction within the orthoferrite. The remaining value of $-10 \mathrm{~T}$ is taken as an intermediate value.

It is seen that, with an increasing ferromagnetic exchange interaction, the time scale of the Co reorientation decreases, where for $J_{\mathrm{c}} m_{0} \sim-150 \mathrm{~T}$, the Co has converged to the reorientation time scale of the orthoferrite [compare to Fig. (1) of Ref. 48]. This may be partially understood by estimating the relaxation time for the Co moment. Inspection of Eqs. (9) and (11) reveals that $2 \gamma \alpha_{\mathrm{c}} J_{\mathrm{c}} m_{0} D / J$ has units of inverse time and corresponds to the relaxation time of the damped LLG dynamics. Using the parameters of Fig. 11, the corresponding relaxation times are approximately $120,1.2$, and $0.08 \mathrm{~ns}$ for $J_{\mathrm{c}} m_{0}$ corresponding, respectively, to $-0.1,-10$, and $-150 \mathrm{~T}$. For the case of $J_{\mathrm{c}} m_{0}$ equal to -10 and $-150 \mathrm{~T}$, these time scales are quite compatible with the relaxation entailed in Fig. 11, however, for the case of $J_{\mathrm{c}} m_{0}=-0.1 \mathrm{~T}$ it is clearly an overestimate. This is most likely due to the now-dominant demagnetization field of $1.8 \mathrm{~T}$ arising from the thin-film anisotropy which results in little out-of-plane dynamics of the 
Co moment, a contribution that has not been included in the above time estimate. It is in this regime, with $J_{\mathrm{c}} m_{0}=-0.1 \mathrm{~T}$ that this simple model reproduces quite well the measured Co reorientation of $4^{\circ}$ as shown in Fig. 3 in Sec. III as continuous lines, including the initial overshot which corresponds to a damped spin precession. It would be tempting to push the comparison further; however, one should keep in mind that the model used is rather simple and that the $\mathrm{SmFeO}_{3}$ dynamics is more complex than initially anticipated.

\section{CONCLUSIONS}

Ultrafast-laser-induced spin reorientation in a $\mathrm{Co} / \mathrm{SmFeO}_{3}$ heterostructure was investigated employing time-resolved XMCD PEEM imaging techniques. It is found that, subsequent to the laser-induced heat pulse, the Co spin direction changes within $100 \mathrm{ps}$ to a new orientation under the influence of the orthoferrite substrate. However, the amount of change that can be obtained in these experiments is at most $10^{\circ}$ compared to the $90^{\circ}$ achievable in the static case. Simulations of the heat profile induced in the heterostructure and of the resulting equilibrium spin configuration in the orthoferrite substrate done by considering the competition between the exchange and anisotropy energy, and comparison of these simulations with the experiments suggest that the dynamics of the reorientation in the $\mathrm{SmFeO}_{3}$ is more complex than that driven by an adiabatic heating. Single-shot time-resolved measurement in different orthoferrites showing the SRT could give insight into the effect responsible for the limited laserinduced reorientation. Nevertheless, fast laser control of a ferromagnet via an antiferromagnet is demonstrated in this system without apparent loss of coupling between the two layers.

\section{ACKNOWLEDGMENTS}

This work was partially supported by de Nederlandse Organisatie voor Wetenschappelijk Onderzoek (NWO), NanoSci-E+ program, Foundation for Fundamental Research (FOM) and the Technology Foundation (STW), the European Union's Seventh Framework Programme (FP7/2007-2013) Grants No. NMP3-SL-2008-214469 (UltraMagnetron) and No. 214810 (FANTOMAS), as well as the European Research Council (FP7/2007-2013)/ERC Grant No. 257280 (Femtomagnetism). Part of this work was performed at the Swiss Light Source, Paul Scherrer Institut, Villigen, Switzerland. We thank C. Milne for the use of the Duetto laser, A. Bullemer and M. Horisberger for the sample preparation, and A. Steger and J. Honegger for their support. R. V. Pisarev thanks RFBR for financial support.
*Present address: Helmholtz-Zentrum Berlin für Materialien und Energie GmbH, BESSY II, Albert-Einstein-Strasse 15, 12489 Berlin, Germany; loic.le_guyader@helmholtz-berlin.de

${ }^{1}$ E. Beaurepaire, J.-C. Merle, A. Daunois, and J.-Y. Bigot, Phys. Rev. Lett. 76, 4250 (1996).

${ }^{2}$ M. Battiato, K. Carva, and P. M. Oppeneer, Phys. Rev. Lett. 105, 027203 (2010).

${ }^{3}$ A. Kirilyuk, A. V. Kimel, and Th. Rasing, Rev. Mod. Phys. 82, 2731 (2010).

${ }^{4}$ L. D. Landau, E. M. Lifshitz, and L. P. Pitaevskii, Electrodynamics of Continuous Media (Butterworth-Heinemann, Oxford, 1984).

${ }^{5}$ R. L. Stamps, J. Phys. D: Appl. Phys. 33, R247 (2000).

${ }^{6} \mathrm{~F}$. Radu and H. Zabel, in Magnetic Heterostructures, Springer Tracts in Modern Physics, Vol. 227, edited by H. Zabel and S. Bader (Springer, Berlin, Heidelberg, 2008), pp. 97-184.

${ }^{7}$ M. Fiebig, N. P. Duong, T. Satoh, B. B. V. Aken, K. Miyano, Y. Tomioka, and Y. Tokura, J. Phys. D: Appl. Phys. 41, 164005 (2008).

${ }^{8}$ T. Kampfrath, A. Sell, G. Klatt, A. Pashkin, S. Mahrlein, T. Dekorsy, M. Wolf, M. Fiebig, A. Leitenstorfer, and R. Huber, Nat. Photonics 5, 31 (2011).

${ }^{9}$ K. Holldack, N. Pontius, E. Schierle, T. Kachel, V. Soltwisch, R. Mitzner, T. Quast, G. Springholz, and E. Weschke, Appl. Phys. Lett. 97, 062502 (2010).

${ }^{10}$ S. L. Johnson, R. A. de Souza, U. Staub, P. Beaud, E. MöhrVorobeva, G. Ingold, A. Caviezel, V. Scagnoli, W. F. Schlotter, J. J. Turner et al., Phys. Rev. Lett. 108, 037203 (2012).

${ }^{11}$ G. Ju, A. V. Nurmikko, R. F. C. Farrow, R. F. Marks, M. J. Carey, and B. A. Gurney, Phys. Rev. B 58, R11857 (1998).

${ }^{12}$ G. Ju, A. V. Nurmikko, R. F. C. Farrow, R. F. Marks, M. J. Carey, and B. A. Gurney, Phys. Rev. Lett. 82, 3705 (1999).
${ }^{13}$ G. Ju, L. Chen, A. V. Nurmikko, R. F. C. Farrow, R. F. Marks, M. J. Carey, and B. A. Gurney, Phys. Rev. B 62, 1171 (2000).

${ }^{14}$ M. C. Weber, H. Nembach, and J. Fassbender, J. Appl. Phys. 95, 6613 (2004).

${ }^{15}$ M. C. Weber, H. Nembach, B. Hillebrands, and J. Fassbender, J. Appl. Phys. 97, 10A701 (2005).

${ }^{16}$ F. Dalla Longa, J. T. Kohlhepp, W. J. M. de Jonge, and B. Koopmans, J. Appl. Phys. 103, 07B101 (2008).

${ }^{17}$ F. Dalla Longa, J. T. Kohlhepp, W. J. M. de Jonge, and B. Koopmans, Phys. Rev. B 81, 094435 (2010).

${ }^{18}$ K. A. Seu and A. C. Reilly, J. Appl. Phys. 103, 07C104 (2008).

${ }^{19}$ A. Porat, S. Bar-Ad, and I. K. Schuller, Europhys. Lett. 87, 67001 (2009).

${ }^{20}$ L. Joly, F. Nolting, A. V. Kimel, A. Kirilyuk, R. V. Pisarev, and Th. Rasing, J. Phys.: Condens. Matter 21, 446004 (2009).

${ }^{21}$ S. M. Shapiro, J. D. Axe, and J. P. Remeika, Phys. Rev. B 10, 2014 (1974).

${ }^{22}$ D. Treves, J. Appl. Phys. 36, 1033 (1965).

${ }^{23}$ R. M. White, R. J. Nemanich, and C. Herring, Phys. Rev. B 25, 1822 (1982).

${ }^{24}$ J.-H. Lee, Y. K. Jeong, J. H. Park, M.-A. Oak, H. M. Jang, J. Y. Son, and J. F. Scott, Phys. Rev. Lett. 107, 117201 (2011).

${ }^{25}$ W. Gil, D. Görlitz, M. Horisberger, and J. Kötzler, Phys. Rev. B 72, 134401 (2005)

${ }^{26}$ M. Abe, K. Kaneta, M. Gomi, Y. Mori, and S. Nomura, Jpn. J. Appl. Phys. 16, 1799 (1977).

${ }^{27}$ R. M. Bozorth, Phys. Rev. 96, 311 (1954).

${ }^{28}$ E. Klokholm and J. Aboaf, J. Appl. Phys. 53, 2661 (1982).

${ }^{29}$ M. Matsuoka, M. Naoe, and Y. Hoshii, Electron. Commun. Jpn. (Part II: Electron.) 69, 1 (1986). 
${ }^{30}$ U. Flechsig, F. Nolting, A. F. Rodríguez, J. Krempaský, C. Quitmann, T. Schmidt, S. Spielmann, and D. Zimoch, AIP Conf. Proc. 1234, 319 (2010)

${ }^{31}$ A. Scholl, H. Ohldag, F. Nolting, J. Stöhr, and H. A. Padmore, Rev. Sci. Instrum. 73, 1362 (2002).

${ }^{32}$ L. Le Guyader, A. Kleibert, A. Fraile Rodríguez, S. El Moussaoui, A. Balan, M. Buzzi, J. Raabe, and F. Nolting, J. Electron. Spectrosc. Relat. Phenom. 185, 371 (2012).

${ }^{33}$ A. Mikkelsen, J. Schwenke, T. Fordell, G. Luo, K. Klunder, E. Hilner, N. Anttu, A. A. Zakharov, E. Lundgren, J. Mauritsson et al., Rev. Sci. Instrum. 80, 123703 (2009).

${ }^{34}$ N. M. Buckanie, J. Göhre, P. Zhou, D. von der Linde, M. Horn-von Hoegen, and F.-J. Meyer zu Heringdorf, J. Phys.: Condens. Matter 21, 314003 (2009).

${ }^{35}$ J. A. de Jong, I. Razdolski, A. M. Kalashnikova, R. V. Pisarev, A. M. Balbashov, A. Kirilyuk, Th. Rasing, and A. V. Kimel, Phys. Rev. Lett. 108, 157601 (2012).

${ }^{36}$ K. Ohta and H. Ishida, Appl. Opt. 29, 2466 (1990).

${ }^{37}$ The depth $y$ starts at the Pt surface with the value of $1 \mathrm{~nm}$ instead of $0 \mathrm{~nm}$ to avoid the logarithm singularity.

${ }^{38}$ P. A. Usachev, R. V. Pisarev, A. M. Balbashov, A. V. Kimel, A. Kirilyuk, and Th. Rasing, Phys. Solid State 47, 2292 (2005).

${ }^{39}$ K. P. Belov, A. K. Zvezdin, A. M. Kadomtseva, and R. Z. Levitin, Sov. Phys. Usp. 19, 574 (1976).

${ }^{40}$ There exist an apparent discrepancy in the literature concerning the $K_{2}$ and $K_{4}$ values. The one given in Ref. 49, which is based on Ref. 50, appears to be $10^{3}$ times smaller than in Refs. 51 and 52. In fact, Fig. 5 of Ref. 50 can only be reproduced with $10^{3}$ times larger values, suggesting the presence of a typo in that original paper. For the simulations, we used the values given in Ref. 51.

${ }^{41}$ S. Geller and E. A. Wood, Acta Crystallogr. 9, 563 (1956).

${ }^{42}$ P. Bruno, Phys. Rev. Lett. 83, 2425 (1999).

${ }^{43}$ A. V. Kimel, A. Kirilyuk, A. Tsvetkov, R. V. Pisarev, and Th. Rasing, Nature (London) 429, 850 (2004).

${ }^{44}$ J. A. de Jong, A. V. Kimel, R. V. Pisarev, A. Kirilyuk, and Th. Rasing, Phys. Rev. B 84, 104421 (2011).

${ }^{45}$ A. V. Kimel, B. A. Ivanov, R. V. Pisarev, P. A. Usachev, A. Kirilyuk, and Th. Rasing, Nat. Phys. 5, 727 (2009).

${ }^{46}$ N. Pontius, T. Kachel, C. S. ler Langeheine, W. F. Schlotter, M. Beye, F. Sorgenfrei, C. F. Chang, A. Föhlisch, W. Wurth, P. Metcalf, I. Leonov, A. Yaresko, N. Stojanovic, M. Berglund, N. Guerassimova, S. Düsterer, H. Redlin, and H. A. Dürr, Appl. Phys. Lett. 98, 182504 (2011).

${ }^{47}$ V. G. Bar'yakhtar, M. V. Chetkin, B. A. Ivanov, and S. N. Gadetskii, Dynamics of Topological Magnetic Solitons: Experiment and Theory (Springer-Verlag, Berlin, 1994).

${ }^{48}$ See Supplemental Material at http://link.aps.org/supplemental/ 10.1103/PhysRevB.87.054437 for a derivation of the "Pendulum approach" applied to the $\mathrm{SmFeO}_{3}$ system.

${ }^{49}$ H. P. J. Wijn, in Landolt-Börnstein - Group III Condensed Matter, Vol. 27F3, edited by H. P. J. Wijn (Springer, Berlin, Heidelberg, 1994).

${ }^{50}$ M. Abe, M. Gomi, K. Shōno, Y. Mori, and S. Nomura, Jpn. J. Appl. Phys. 16, 279 (1977).

${ }^{51}$ S. Kagoshima and S. Takayama, J. Phys. Soc. Jpn. 29, 793 (1970).

${ }^{52}$ K. P. Belov, R. A. Volkov, B. P. Goranskii, A. M. Kadomtseva, and V. V. Uskov, Sov. Phys. Solid State 11, 935 (1969). 